\title{
Well-defined and stable nanomicelles self-assembled from brush cyclic and tadpole copolymer amphiphiles: a versatile smart carrier platform
}

\author{
Brian J Ree ${ }^{1,2,5}$, Yusuke Satoh ${ }^{2,5}$, Kyeong Sik Jin ${ }^{3}$, Takuya Isono ${ }^{2}$, Won Jong Kim ${ }^{1}$, Toyoji Kakuchi ${ }^{2}$, \\ Toshifumi Satoh ${ }^{2}$ and Moonhor Ree ${ }^{3,4}$
}

This study reports the first well-defined and stable nanomicelles $(20-30 \mathrm{~nm}$ in diameter) self-assembled from amphiphilic brush (comb-like) cyclic and tadpole-shaped copolymers composed of hydrophobic n-decyl and hydrophilic 2-(2-methoxyethoxy)ethoxy) ethyl bristle blocks based on a poly(glycidyl ether) backbone. The micelle formation behaviour and structural details were investigated by synchrotron X-ray scattering analysis in a rigorous and complementary manner. The amphiphilic brush cyclic topology facilitates more compact and stable aggregation behaviour in the micelle core and a more densely packed corona, which prevents intermicellar aggregation. The presence of a hydrophobic component with brush cyclic topology inside the core is identified as the primary micelle stabilizing factor, enabling stable core aggregation and sharper core-corona interface formation. The presence of a hydrophilic brush cyclic component in the corona is determined as the secondary micelle stabilizing factor, helping nullify the corona penetration by polymer chains from other micelles and ultimately prevent the intermicellar aggregation-mediated collapse of the micellar structure. Overall, the brush cyclic topology was confirmed to be beneficial for forming highly stable nanomicelles with an extremely narrow (pseudo-monodisperse) size distribution compared with conventional linear topology and tadpole topologies. All the results of this study provide a unique opportunity for designing advanced functional high-performance amphiphilic materials for nanomicelles that are unattainable by other conventional methods and broadening their applications in various fields, including drug delivery, biomedical imaging, foods, cosmetics, smart coatings, photonics and molecular electronics.

NPG Asia Materials (2017) 9, e453; doi:10.1038/am.2017.205; published online 8 December 2017

\section{INTRODUCTION}

Molecular topology has been a key aspect of numerous research topics in polymer science because of its impact on the physical properties of polymers. ${ }^{1-5}$ In particular, the emergence of various cyclic topologies based on linear polymers has recently drawn great attention from academia because of the unique traits of these systems, such as increased glass transition temperature, lower viscosity and smaller hydrodynamic radius due to the lack of a chain end group effect. ${ }^{1-10}$ In addition, cyclic block copolymers have received interest due to selfassembly characteristics that deviate from the typical behaviours of their linear analogues. ${ }^{3,5,11-24}$
In the case of micelle formation in solution, cyclic block copolymers were reported to form relatively more compact micelles than their linear analogues. ${ }^{11-13,15,16,18,23}$ The cyclic block copolymer micelles, however, were very often evaluated qualitatively. ${ }^{11,15,18,22,23}$ Their stabilities were relatively higher than those of their linear analogues but still limited to a certain extent. ${ }^{1,11,13,15,18,22,23}$ Thus, they have not yet been fully understood in the aspect of structural characteristics (shape, size, size distribution, structural components, density gradient, stability and so on). In fact, micelles have great potent for applications as a carrier system in various fields, such as drug delivery, biomedical imaging, foods, cosmetics, coatings, photonics and molecular

\footnotetext{
${ }^{1}$ Department of Chemistry, Pohang University of Science and Technology (POSTECH), and Center for Self-assembly and Complexity, Institute for Basic Science, Pohang, Republic of Korea; ${ }^{2}$ Faculty of Engineering, Graduate School of Chemical Sciences and Engineering, Hokkaido University, Sapporo, Japan; ${ }^{3}$ Pohang Accelerator Laboratory, Pohang University of Science and Technology (POSTECH), Pohang, Republic of Korea and ${ }^{4}$ Department of Chemistry, Division of Advanced Materials Science, and Polymer Research Institute, Pohang University of Science and Technology (POSTECH), Pohang, Republic of Korea

5These authors contributed equally to this work.

Correspondence: Professor W Kim, Department of Chemistry, Pohang University of Science and Technology, and Center for Self-assembly and Complexity, Institute for Basic Science, 77 Cheongam-ro, Hyoja-dong, Nam-gu, Pohang 37673, Republic of Korea.

E-mail: wjkim@postech.ac.kr

or Professor T Satoh, Faculty of Engineering, Graduate School of Chemical Sciences and Engineering, Hokkaido University N13W8, Kita-ku, Sapporo 060-8628, Japan.

E-mail: satoh@eng.hokudai.ac.jp

or Professor M Ree, Department of Chemistry, Division of Advanced Materials Science, and Polymer Research Institute, Pohang University of Science and Technology, 77 Cheongam-ro, Hyoja-dong, Nam-gu, Pohang 37673, Republic of Korea.

E-mail: ree@postech.edu

Received 20 August 2017; revised 21 September 2017; accepted 25 September 2017
} 
electronics. The applicability and performance are highly dependent on the micelle's size, size distribution and stability. In the field of drug delivery, for example, the effectiveness of a micellar drug carrier is determined by how well it can reach the targeted tissue or diseased areas. Despite having a simple criterion, many obstacles hinder the drug delivery efficiency and thus complicate the design of a carrier system that will persist in the circulatory system until its arrival at the target tissue. The obstacles, which are physiological mechanisms for eliminating foreign objects, are directly related to the size and structural stability of the micelle. For instance, micelles smaller than $20 \mathrm{~nm}$ are prone to urinary elimination via passage through the glomerular fenestrations when circulating through the kidney. ${ }^{25}$ On the other hand, micelles larger than $100 \mathrm{~nm}$ are at risk of hepatic elimination via accumulation in the liver, elimination by reticuloendothelial system clearance (that is, macrophage), or accumulation in the spleen and lungs. ${ }^{26}$ In general, micelles with sizes between 20 and $50 \mathrm{~nm}$ can efficiently enter cancerous tissues through the openings of loosely packed cancer cells via the enhanced permeation and retention effect; the rapid growth of cancer lowers the packing density among cancer cells. ${ }^{27,28}$ Thus, the impact of size, size distribution and structural stability is crucial to the overall performance of micelles in different applications. Overall, the development of micelles with a desired size, a unimodal narrow size distribution and structural stability remains in its early stages.

In this study, we aimed to make well-defined and stable micelles with a size of $20-30 \mathrm{~nm}$, a unimodal and extremely narrow size distribution and high structural stability by tailoring the topology and molecular weight of polymer amphiphiles. To achieve this goal, a series of amphiphilic brush (comb-like) diblock copolymers with various topologies (cyclic, tadpole and linear shapes) were synthesized. All the topological copolymers were composed of hydrophobic and hydrophilic bristle blocks based on a poly(glycidyl ether) backbone (Figure 1). The incorporation of hydrophobic bristles is to induce stable and tight packing behaviour within the micelle core, as the

\section{a}

Brush Linear Diblock Copolymer Amphiphile

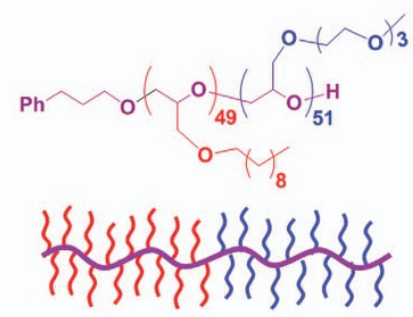

C

Brush Tadpole-A Diblock Copolymer Amphiphile

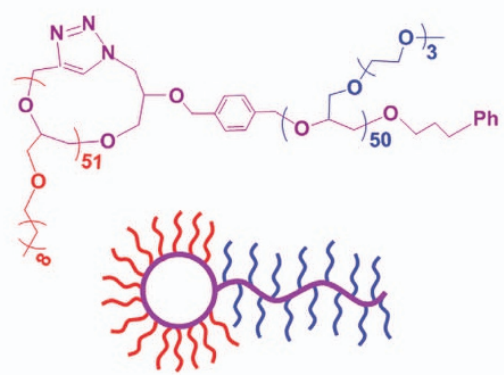

packing ability of the bristles is more favourable and effective than the packing ability of a simple hydrophobic linear polymer backbone due to the greater number of bristles and higher packing density produced by the closely positioned bristles. Similarly, hydrophilic bristles possess the ability to structurally stabilize the micelle corona. In contrast to a simple hydrophilic linear polymer backbone, a hydrophilic brush block has a much greater volume upon solvation, thereby reducing the crevices and their population in the micelle corona exposed to potential penetration by polymer chains from other micelles and ultimately preventing intermicellar aggregation. All topological amphiphiles were confirmed to successfully form micelles in an ethanolwater cosolvent system by synchrotron X-ray scattering analysis. The measured X-ray scattering data were successfully analysed using a new quantitative analysis methodology and corresponding software programme developed in this study, providing structural details of the topological polymer micelles: overall shape, structural components and their dimensions, size and size distribution, electron density profile in the radial direction, aggregation number of polymer chains and blob size. All the brush block copolymer amphiphiles were found to form ellipsoidal micelles with a unimodal size distribution; these micelles consisted of a dense core, a dense corona and a solvated corona. However, the micelles exhibited significantly different structural characteristics, stability and size distribution depending on the brush block copolymer topologies. The brush cyclic block copolymer formed the most compact and stable nanomicelles with an extremely narrow (pseudo-monodisperse) size distribution compared to the other topologies. Overall, the amphiphilic brush cyclic topology had a significant impact on the reduction of micelle size, the narrowing of size distribution and the enhancement of micelle stability.

\section{MATERIALS AND METHODS}

Materials and micelle preparation

All diblock copolymers in this study were composed of poly $n$-decyl glycidyl ether) (PDGE) and poly(2-(2-(2-methoxyethoxy)ethoxy)ethyl glycidyl ether) (PTEGGE) blocks with the degrees of polymerization tabulated in Table 1.

\section{b}

Brush Cyclic Diblock Copolymer Amphiphile
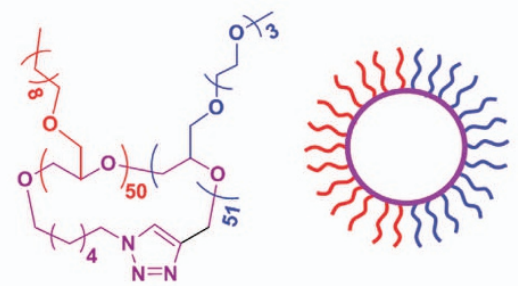

d

Brush Tadpole-B Diblock Copolymer Amphiphile

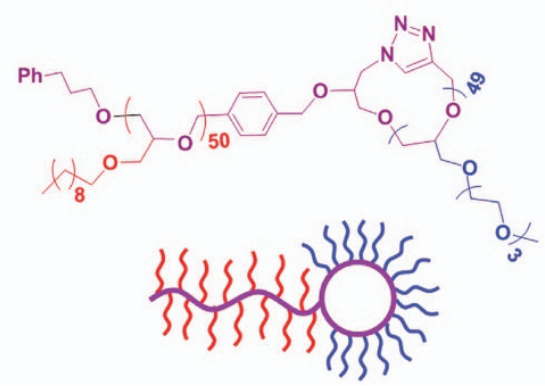

Figure 1 Chemical structures of the amphiphilic brush diblock copolymers with various topologies investigated in this study. 
Table 1 Molecular characteristics, degree of polymerization and volume fractions of brush polymers with various topologies

\begin{tabular}{|c|c|c|c|c|c|c|c|c|}
\hline \multirow{2}{*}{ Polymer } & \multirow{2}{*}{$M_{\mathrm{n}, \mathrm{NMR}}{ }^{a}\left(\mathrm{~g} \mathrm{~mol}^{-1}\right)$} & \multirow{2}{*}{$M_{\mathrm{w}} / M_{\mathrm{n}}{ }^{b}$} & \multirow{2}{*}{$\rho_{\mathrm{e}}^{c}\left(n m^{-3}\right)$} & \multirow{2}{*}{$\rho_{\mathrm{m}}{ }^{d}\left(\mathrm{~g} \mathrm{~cm}^{-3}\right)$} & \multicolumn{2}{|c|}{ PDGE block } & \multicolumn{2}{|c|}{ PTEGGE block } \\
\hline & & & & & $D P_{\mathrm{PDGE}}^{e}$ & $\phi_{\mathrm{PDGE}}^{f}$ & $D P_{\text {PTEGGE }} g$ & $\phi_{\text {PTEGGE }}{ }^{h}$ \\
\hline \multicolumn{9}{|c|}{ Amphiphilic brush diblock copolymer } \\
\hline Linear & 21900 & 1.04 & & & 49 & 0.49 & 51 & 0.51 \\
\hline Cyclic & 22300 & 1.04 & & & 50 & 0.52 & 51 & 0.48 \\
\hline Tadpole-A & 22200 & 1.04 & & & 50 & 0.52 & 51 & 0.48 \\
\hline Tadpole-B & 21700 & 1.04 & & & 50 & 0.49 & 49 & $0.51^{i}$ \\
\hline \multicolumn{9}{|l|}{ Brush homopolymer } \\
\hline Linear PDGE & 11100 & 1.03 & 341 & 1.01 & & & & \\
\hline Cyclic PDGE & 11000 & 1.02 & 310 & 0.92 & & & & \\
\hline Linear PTEGGE & 11200 & 1.04 & 353 & 1.05 & & & & \\
\hline
\end{tabular}

Abbreviations: DP, degree of polymerization; GPC, gel permeation chromatography; ${ }^{1} \mathrm{H}$ NMR, proton nuclear magnetic resonance; PDGE, poly $(n$-decyl glycidyl ether); PDI, polydispersity index;

PTEGGE, poly(2-(2-(2-methoxyethoxy)ethoxy)ethyl glycidyl ether).

a Number-average molecular weight of polymer determined by ${ }^{1} \mathrm{H}$ NMR spectroscopic analysis.

bPDI of polymer determined by GPC analysis in THF.

bPDI of polymer determined by GPC analysis in THF.
cElectron density of polymer in films determined by X-ray reflectivity analysis.

dMass density of polymer in films obtained from the electron density determined by X-ray reflectivity analysis.

eNumber-average degree of polymerization of PDGE block determined by ${ }^{1} \mathrm{H}$ NMR.

fVolume fraction of the PDGE block estimated from the $M_{\mathrm{n}, \mathrm{NMR}}$ and $\rho_{\mathrm{m}}$ data

gNumber-average degree of polymerization of the PTEGGE block determined by ${ }^{1} \mathrm{H}$ NMR.

hVolume fraction of the PTEGGE block estimated from the $M_{\mathrm{n}, \mathrm{NMR}}$ and $\rho_{\mathrm{m}}$ data.

'Volume fraction estimated from the assumption that the $\rho_{\mathrm{m}}$ of the cyclic PTEGGE block is $8.9 \%$ lower than that of the linear analogue, as observed in the linear and cyclic PDGE polymers;

specifically, the cyclic PTEGGE block is assumed to have $\rho_{\mathrm{m}}=0.96 \mathrm{~g} \mathrm{~cm}^{-3}$.

They were prepared according to previously reported synthetic schemes: ${ }^{29}$ for the synthetic methods, see Supplementary Scheme S1 (synthetic details are given in the Supplementary Information). The block copolymers were introduced into various organic solvents and their mixtures to investigate the optimal conditions for micelle formations. For the critical micelle concentration (CMC) measurement of each polymer, a series of micellar solutions was also prepared over the concentration range of $0.001-0.1 \mathrm{wt} \%$.

\section{Synchrotron X-ray scattering measurements}

Solution X-ray scattering measurements were carried out at the $4 \mathrm{C}$ beamline $e^{30-32}$ of the Pohang Accelerator Laboratory at Pohang University of Science and Technology. Each micellar solution was filtered through a disposable syringe equipped with a polytetrafluoroethylene filter with a $0.2 \mu \mathrm{m}$ pore size before measurements. All scattering measurements were carried out with sample-to-detector distances of $4 \mathrm{~m}$ and $1 \mathrm{~m}$ at room temperature. A quartz capillary tube $(1.5 \mathrm{~mm}$ inner diameter $)$ was used as a solution cell; $50 \mu \mathrm{l}$ of polymer solution was loaded in the sample cell. The scattering data were collected through a two-dimensional charge-coupled detector (model Rayonix two-dimensional Mar, Evanston, IL, USA) using an $\mathrm{X}$-ray radiation source $(\lambda=0.0756 \mathrm{~nm}$, wavelength) with an exposure time of $60 \mathrm{~s}$. The scattering angle was calibrated with precalibrated polystyrene- $b$ polyethylene- $b$-polybutadiene- $b$-polystyrene block copolymer and silver behenate powder (Tokyo Chemical Industry, Tokyo, Japan). The two-dimensional scattering data were circularly averaged with respect to the beam centre and normalized to the intensity of the transmitted X-ray beam monitored via a scintillation counter positioned behind the sample. The scattering data were further corrected for the scattering due to the solvent.

\section{Synchrotron X-ray reflectivity measurements}

Synchrotron X-ray reflectivity measurements were conducted at the 3D beamline $^{33}$ of Pohang Accelerator Laboratory. Polymer thin films were prepared on silicon substrates via conventional spin coating and drying under vacuum at room temperature for 1 day; the individual polymers were dissolved in ethanol and filtered with polytetrafluoroethylene membrane filters $(0.2 \mu \mathrm{m}$ pore size). The wavelength of the incident $\mathrm{X}$-ray beam was tuned to $\lambda=0.1541 \mathrm{~nm}$ by using a $\mathrm{Si}(111)$ double-crystal monochromator. The incident $\mathrm{X}$-ray beam was collimated at the sample position to $2 \mathrm{~mm}$ (horizontal) by $0.1 \mathrm{~mm}$ (vertical). The reflected intensity was normalized to the intensity of the primary beam monitored through an ionization chamber. The specular reflection was measured in $\theta-2 \theta$ scanning mode and $2 \theta$ was scanned at increments of $0.01^{\circ}$ at angles $<1.0^{\circ}$ for determining the critical angles of the silicon substrate and of the polymer film. At the high angles, the step width was increased to $0.02-0.1^{\circ}$. The obtained reflectivity data were subjected to a geometrical correction and background subtraction procedure described in the literature. ${ }^{33}$ The measured X-ray reflectivity data were quantitatively analysed with a recursive formula given by the Parratt dynamic theory. ${ }^{33,34}$

\section{RESULTS AND DISCUSSION}

Cyclic, tadpole and linear diblock copolymers of two monomers, $n$ decyl glycidyl ether and 2-(2-(2-methoxyethoxy)ethoxy)ethyl glycidyl ether, were synthesized and characterized as described in the Supplementary Information. As summarized in Table 1, the PDGE and PTEGGE blocks in all brush copolymers were well controlled to have an equivalent degree of polymerization of $50( \pm 1)$. Their overall number-average molecular weights $\left(M_{\mathrm{n}, \mathrm{NMR}}\right)$ were determined to be in the range of $21700-22300$ by proton nuclear magnetic resonance spectroscopic analysis. The polydispersity indices (PDI) were determined to be 1.04 by using gel permeation chromatography analysis. In addition, linear and cyclic homopolymers were synthesized in a similar controlled manner to the block copolymers: $M_{\mathrm{n}, \mathrm{NMR}}=11000 \sim 11200$ and $\mathrm{PDI}=1.02 \sim 1.04$. Overall, well-defined topological copolymers and homopolymers were obtained.

For the individual topological copolymers, micellization was attempted in various organic solvents and their mixtures. The brush cyclic and tadpole copolymers were found to successfully form micelles in a cosolvent system with a composition of $75 \mathrm{wt} \%$ ethanol and $25 \mathrm{wt} \%$ deionized water. Their linear analogue also formed micelles under the same conditions. The micellar solutions of each copolymer were prepared by initial solvation in ethanol and subsequent addition of deionized water. The CMC was determined by measuring X-ray scattering data with different solution concentrations. The measured scattering data were converted to the invariant $Q$ values and, by plotting $Q$ against the corresponding polymer concentration values, the $\mathrm{CMC}$ was obtained; a representative of the $\mathrm{CMC}$ determination procedure is shown in Supplementary Figure S1. The brush cyclic and tadpole copolymers exhibited CMC values in the 
range from 0.015 to $0.026 \mathrm{wt} \%$ depending on the molecular topology, as listed in Table 2 . These CMC values were surprisingly smaller than that $(0.028 \mathrm{wt} \%)$ of the linear analogue.

For the micelle solutions with a polymer concentration of $0.5 \mathrm{wt} \%$, $\mathrm{X}$-ray scattering measurements were carried out at room temperature. Representatives of the measured X-ray scattering data are shown in Figure 2.

The X-ray scattering data were initially analysed by using Guinier's $\mathrm{law}^{35}$ and the indirect Fourier transformation (IFT) method, ${ }^{36}$ which are model independent methods; these analytical schemes are described in the Supplementary Information. The Guinier analysis (a model independent method) provides the radius of gyration $R_{\mathrm{g}, \mathrm{G}}$ of micelles in solution. A representative of the Guinier analysis results is given in Supplementary Figure S2 and the obtained results are summarized in Table 2. The analysis shows a decreasing trend in micelle size for the brush linear copolymer, brush tadpole-B copolymer, brush tadpole-A copolymer and brush cyclic copolymer. This trend is confirmed by the IFT analysis, which extracts structural information, such as radius of gyration $R_{\mathrm{g}, \mathrm{IFT}}$ and maximum dimension $D_{\max }$ (that is, the diameter of micelle), directly from the scattering profile (Table 2). The $R_{\mathrm{g}}$ values obtained from these two analysis methods, however, agree to within only a few nanometers $(0.41-1.61 \mathrm{~nm})$. The disagreement between the two methods may be due to the limitation of Guinier's law, in which the error in $R_{\mathrm{g}, \mathrm{G}}$ increases as the shape of the scatterer deviates from an ideal sphere. ${ }^{21}$ In contrast, the IFT analysis bypasses the use of a parameterized model to reduce the impact of missing data and errors in the extracted structural information. Thus, the magnitude of the differences in the $R_{\mathrm{g}}$ values can be used to qualitatively evaluate the shapes of the micelles, with the brush cyclic copolymer micelle taking the shape closest to an ideal sphere and the micelles of the brush linear copolymer and the brush tadpole copolymers showing signs of distortion in their shape (that is, ellipsoids). In addition, the pair distance distribution function $p(r)$ derived from the IFT analysis is another indicator for determining the shapes of micelles. The $p(r)$ functions of the individual micelle systems are plotted in Figure 3a, generally revealing the shape of a bell curve with varying positive skewness. Given that the $p(r)$ function of an ideal sphere is a perfect bell curve, the shapes of all micelles are established as ellipsoids with varying levels of distortion. The extent of distortion in ellipsoidal dimensions can be examined with the quotient of $R_{\max } / R_{\mathrm{g}, \mathrm{IFT}}$ for each micelle. For an ideal homogeneous sphere, its $p(r)$ function displays a peak maximum at the radius of the sphere, $R_{\max }=1.36 R_{\mathrm{g}, \mathrm{IFT} .} \cdot{ }^{21}$ Hence, the quotients of $R_{\max } / R_{\mathrm{g}, \mathrm{IFT}}$ for the individual micellar systems

Table 2 Structural parameters of micelles of the amphiphilic brush diblock copolymers with various topologies obtained from the scattering invariant analysis, Guinier analysis and indirect Fourier transform analysis

\begin{tabular}{|c|c|c|c|c|c|c|}
\hline $\begin{array}{l}\text { Amphiphilic brush diblock } \\
\text { copolymer }\end{array}$ & $\begin{array}{l}C M C^{a} \\
(w t \%)\end{array}$ & $\begin{array}{l}R_{\mathrm{g}, \mathrm{G}^{\mathrm{b}}} \\
(\mathrm{nm})\end{array}$ & $\begin{array}{l}D_{\max }{ }^{\mathrm{c}} \\
(n m)\end{array}$ & $\begin{array}{l}R_{\mathrm{g}, \mathrm{IFT}}{ }^{\mathrm{d}} \\
(\mathrm{nm})\end{array}$ & $\begin{array}{l}R_{\max } \mathrm{e}^{\mathrm{e}} \\
(\mathrm{nm})\end{array}$ & $\begin{array}{l}R_{\max } / \\
R_{\mathrm{g}, \mathrm{IFT}}\end{array}$ \\
\hline Linear & 0.028 & 18.57 & 59.00 & 18.05 & 22.42 & 1.24 \\
\hline Cyclic & 0.026 & 7.75 & 23.00 & 7.97 & 10.58 & 1.33 \\
\hline Tadpole-A & 0.015 & 8.83 & 28.50 & 9.24 & 11.70 & 1.27 \\
\hline Tadpole-B & 0.017 & 9.92 & 31.70 & 11.53 & 12.48 & 1.08 \\
\hline
\end{tabular}

Abbreviations: CMC, critical micelle concentration; IFT, indirect Fourier transformation. ${ }^{a}$ Critical micelle concentration determined by $\mathrm{X}$-ray scattering invariant analysis.

${ }^{b}$ Radius of gyration of micelle determined by Guinier analysis.

cMaximum dimension (that is, diameter) of micelle determined from the $p(r)$ function.

dRadius of gyration of micelle determined by IFT analysis.

eRadius of micelle determined from the peak maximum of the $p(r)$ function. describe that the brush cyclic copolymer forms a slightly distorted ellipsoidal micelle with a quotient value of 1.33 , whereas the brush linear, tadpole-A and tadpole-B analogues show greater distortion in their dimensions with quotient values ranging from 1.08 to 1.27 (Table 2). Furthermore, the deviations in the quotient values from 1.36 indicate that all brush copolymer micelles are in fact inhomogeneous systems, which is consistent with the presence of two micellar structural regions (core and corona). The radial electron density distribution functions $\rho(r)$ derived from the $p(r)$ functions reflect the inhomogeneous nature of the micelles, where each density profile can be roughly divided into a dense core and a solvated corona in which the density decreases in a gradient (Figure 3b). It is shown that each micelle possesses morphological properties unique to the topology of its corresponding brush copolymer, but the Guinier and IFT analyses do not provide specific, quantitative details regarding the structural dimensions of the micelles and the micellization behaviour of the brush copolymers.

Further analysis of the X-ray scattering data were attempted by using two model-dependent methods from the literature: (i) spherical copolymer micelle model analysis ${ }^{13,37,38}$ and (ii) spherical core-fuzzy shell model method ${ }^{13,39}$ (the analytical schemes are described in the Supplementary Information). With these methods, however, the X-ray scattering data could not be analysed well; in particular, the scattering data in the region of $q=0.13 \sim 0.60 \mathrm{~nm}^{-1}$ could not be fitted with the analytical schemes (for a representative of the data analysis results, see Supplementary Figure S3). Overall, the X-ray scattering data analyses with these analytical schemes could provide structural information about micelles in a qualitative manner rather than a quantitative manner.

To extract specific morphological details of micelles in a quantitative manner, we developed a new three phase ellipsoid model based on the principle scattering of a spheroid (that is, an ellipsoid of gyration) and blob form factors, as described in the Supplementary Information. As shown in Figure 4, all X-ray scattering data have been satisfactorily fitted with this new model. The detailed structural parameters of the individual micelle systems are summarized in Table 3 . In addition to the morphological structural parameters, the size distributions of the micelle systems were also extracted through this analysis (Figure 5).

The brush linear diblock copolymer micelle is characterized by a relatively large prolate ellipsoid geometry with $R_{\mathrm{e} \text {,micelle }}=23.00 \mathrm{~nm}$ (micelle radius in the equatorial direction) and $\varepsilon=1.50\left(=R_{\mathrm{p}, \text { micelle }} /\right.$

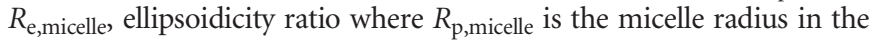
polar direction). The micelle is composed of a core, a dense corona and a solvated corona. The core part has a radius, $r_{\text {core }}=11.60 \mathrm{~nm}$, including a fuzzy component of $2.00 \mathrm{~nm}\left(=t_{\mathrm{f} \text {, core }}\right.$, namely, the fuzzy interfacial layer thickness) in contact with the dense corona part. This core radius is roughly half of the micelle radius. The dense corona has a thickness, $t_{\mathrm{d}, \text { corona }}=6.8 \mathrm{~nm}$ including $t_{\mathrm{f} \text {,d.corona }}=2.30 \mathrm{~nm}$ (the thickness of the fuzzy interfacial layer in contact with the solvated corona), whereas the solvated corona has a thickness, $t_{\mathrm{s}, \text { corona }}=4.60 \mathrm{~nm}$ including $t_{\mathrm{f}, \mathrm{s.corona}}=2.00 \mathrm{~nm}$ (the thickness of the fuzzy interfacial layer in contact with the solvent). The overall corona is further characterized by an average correlation length $\xi$ of $1.50 \mathrm{~nm}$, which describes the average length of the density fluctuations within the polymer chains present in the corona regions. For the micelle, the aggregation number $N_{\mathrm{agg}}$ of the brush linear copolymer chains was additionally estimated to be 557 from the total mass of the hydrophobic PDGE block chains occupying the core volume and an assumption that the core has the same mass density $\rho_{\mathrm{m}}$ as that $\left(1.05 \mathrm{~g} \mathrm{~cm}^{-3}\right)$ of the corresponding homopolymer; here, the $\rho_{\mathrm{m}}$ value of the PDGE homopolymer was obtained from the electron density $\rho_{\mathrm{e}}$ 


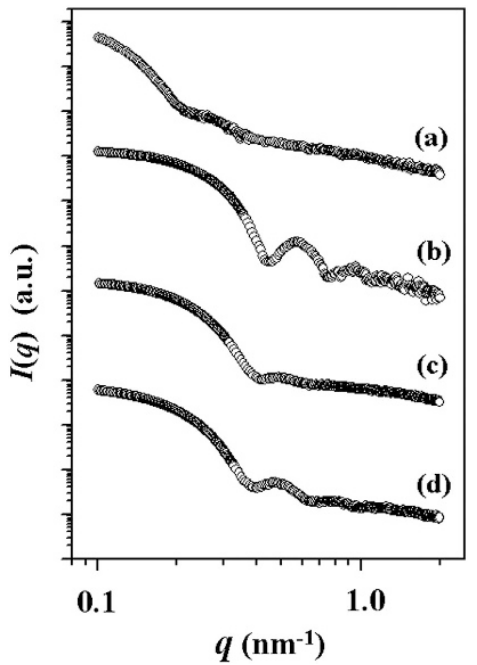

Figure 2 Representative X-ray scattering profiles of the micelles of amphiphilic brush diblock copolymers with various topologies formed in an ethanol/water mixture (75/25 wt\%), which were measured at room temperature: (a) brush linear copolymer; (b) brush cyclic copolymer; (c) brush tadpole-A copolymer; (d) brush tadpole-B copolymer.

a
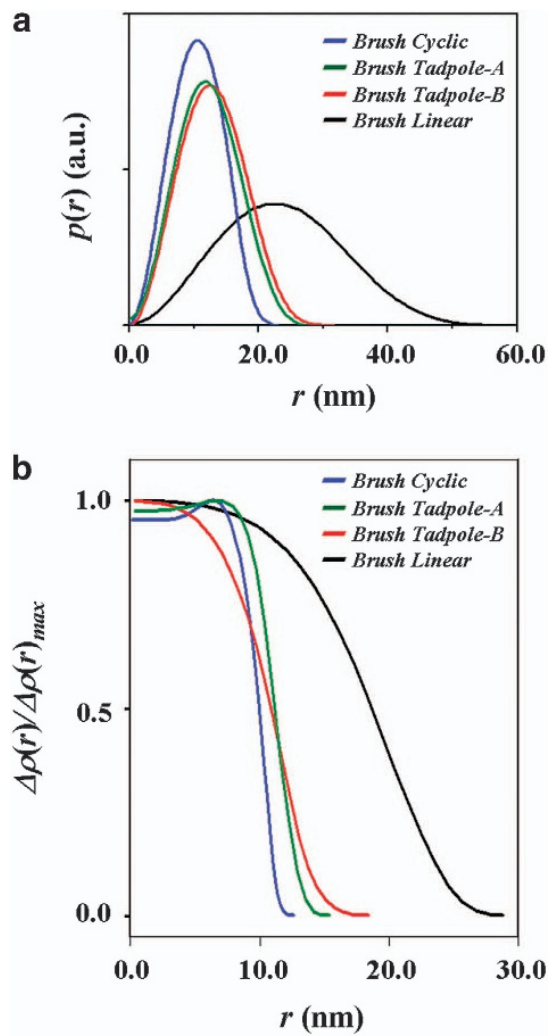

Figure 3 (a) Pair distance distribution functions $p(r)$ and (b) radial density distribution functions $\rho(r)$ obtained from the IFT analysis of the X-ray scattering data in Figure 2: (blue line) brush cyclic copolymer micelle; (green line) brush tadpole-A copolymer micelle; (red line) brush tadpole-B copolymer micelle; (black line) brush linear copolymer micelle.

value $\left(=341 \mathrm{~nm}^{-3}\right)$ determined by synchrotron X-ray reflectivity analysis (Table 1, Supplementary Table S1 and Supplementary Figure S4). Moreover, the micelle exhibits a unimodal size distribution. However, the size distribution is very broad: the micelle radius varies over the range of $16.9-29.1 \mathrm{~nm}$.

The brush cyclic and tadpole copolymer analogues also demonstrate the formation of micelles with a unimodal size distribution. The micelles were also found to consist of a core, a dense corona and a solvated corona. However, the micelles exhibit significantly different structural characteristics as follows.

First, the brush cyclic copolymer forms the most compact micelle with the narrowest size distribution. The micelle size is only half of that of the brush linear copolymer micelle, even though the degrees of polymerization (that is, molecular weights) are almost the same. In addition, the core radius is almost the same as the overall corona thickness. Interestingly, the brush cyclic copolymer micelle is an oblate ellipsoid $(\varepsilon=0.84)$ rather than a prolate ellipsoid. The $\varepsilon$ value is close to the unity, indicating that the micelle is slightly distorted from a spheroid; this distortion level is relatively lower than those of the other micelles. Notably, the micelle is made of only 41 copolymer chains ( $=N_{\mathrm{agg}}$ ); this $N_{\mathrm{agg}}$ value is almost 14 times lower than that (557) of the micelle comprising the brush linear copolymer analogue. The micelle is characterized by a very sharp core-dense corona interface $\left(0.40 \mathrm{~nm}\right.$ interfacial thickness $\left.t_{\mathrm{f} \text {,core }}\right)$, which is a key indicator of high stability in the micelle core; this interface is $\sim 82 \%$ thinner than that of the brush linear copolymer micelle. Such a sharp core-dense corona interface signifies a definite phase separation between the hydrophobic and hydrophilic blocks in the micelle. As a result of the high stability, the aggregation behaviour among the hydrophobic blocks would be consistent throughout the micelle population, thereby yielding the narrowest size distribution. Overall, the amphiphilic brush cyclic topology demonstrated a significant and structurally beneficial impact on the reduction of micelle size, the narrowing of size distribution and the enhancement of micelle stability.

Second, the brush tadpole-A copolymer also forms more compact micelles with a narrower size distribution than its linear analogue. This micelle, however, is larger in size and possesses a broader size distribution than the brush cyclic copolymer micelle. Interestingly, the brush tadpole-A copolymer forms an oblate ellipsoidal micelle, as observed in the brush cyclic analogue. However, the distortion level $(\varepsilon=0.78)$ is higher than that $(\varepsilon=0.84)$ of the brush cyclic copolymer micelle. Notably, the core radius is $38 \%$ shorter than the whole corona thickness $\left(=t_{\mathrm{d}, \text { corona }}+t_{\mathrm{s}, \text { corona }}\right)$, which is different from the balanced length scales of the core and corona regions of the brush linear and cyclic copolymer micelles. Moreover, the core radius is smaller than that of the brush cyclic copolymer micelle. Furthermore, the micelle has an exceptionally low $N_{\text {agg }}$ value of 23 , which is $\sim 24$ times lower than that of the brush linear copolymer micelle and roughly half of that of the brush cyclic copolymer micelle. Given that the brush tadpole-A copolymer is a hybrid of brush linear and cyclic copolymers because of its fully cyclic hydrophobic block and linear hydrophilic block, two critical correlations between the value of $N_{\text {agg }}$ and the topology of the hydrophobic block can be deduced: (i) the presence of cyclic topology within the hydrophobic brush block promotes the assembly of a compact micelle core with a chain aggregation number that is considerably lower than that of the linear copolymer analogue and (ii) the fully cyclic topological feature of the hydrophobic block in the brush tadpole-A copolymer facilitates the formation of a much smaller micelle core than that generated from the semi-cyclic hydrophobic block of the brush cyclic block copolymer. Even though the micelle with the smallest core also had the lowest $N_{\text {agg }}$ value, the dense and solvated corona regions formed by the linear hydrophilic block are 1.5 times thicker than that of the corona regions of the cyclic copolymer micelle. In addition, both the core-dense corona and dense 


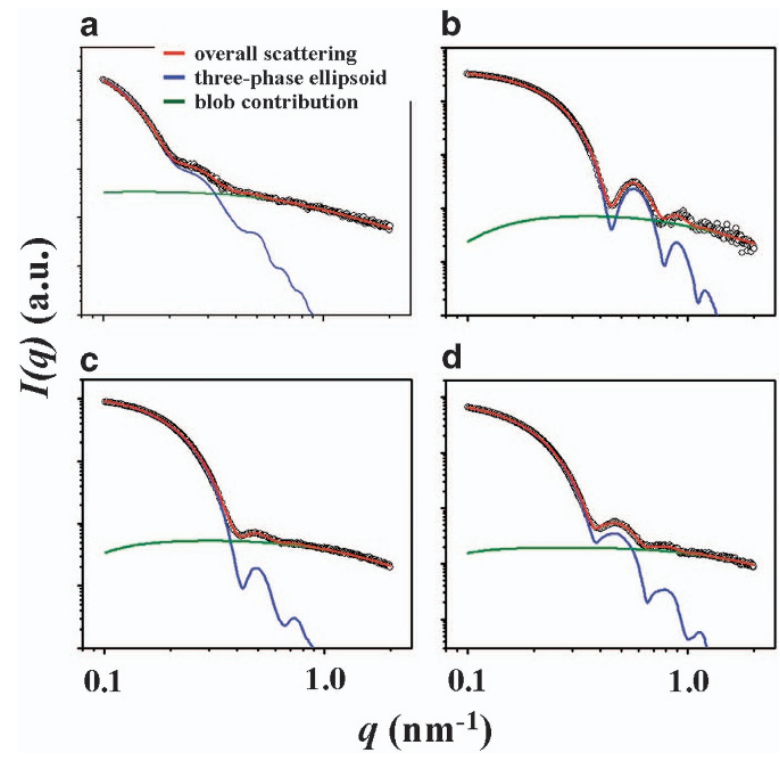

Figure 4 X-ray scattering data analysis results for the micelles of amphiphilic brush diblock copolymers with various topologies formed in an ethanol/water mixture (75/25 wt\%), which were measured at room temperature: (a) brush linear copolymer; (b) brush cyclic copolymer; (c) brush tadpole-A copolymer; (d) brush tadpole-B copolymer. The black symbols are the measured data and the red solid lines represent the sum of the profiles obtained by fitting the data using a three-phase ellipsoid model (blue lines) and blob contributions (green lines).

corona-solvated corona regions exhibit similar interfacial characteristics to those observed in the linear copolymer micelle rather than those observed in the cyclic copolymer micelle. Considering the topology, the formation of thicker corona regions with thicker interfaces can be correlated with the presence of a hydrophilic linear block along with the chain conformation upon solvation. The linear hydrophilic block possesses a backbone chain end that functions by positioning itself at the edge or near the edge of the corona region to maximize the distance from the rest of its own chain and other neighbouring hydrophilic chains for entropic gain and stability. Naturally, this would lead to a loosely stretched chain conformation. In the case of the brush cyclic copolymer, the hydrophilic block is semi-cyclic and without a backbone chain end. This causes the hydrophilic block to adopt a loop-like chain conformation, in which the resulting corona thickness is relatively shorter than that of the corona formed by a linear hydrophilic block. The subsequent outcome of a linear hydrophilic block forming a thicker corona arises from the presence of thicker interfaces, which is predictable because the hydrophilic block chains are in a loosely stretched conformation. This characteristic, however, may have a negative effect on the structural stability of the micelle, as indicated by the relatively broader micelle size distribution than that of the cyclic copolymer micelle. Overall, the stability of the brush tadpole-A copolymer micelle could be lower than that of the brush cyclic copolymer micelle.

Third, the brush tadpole-B copolymer forms a prolate ellipsoidal micelle similar to that observed with the linear analogue and the micelle's dimensions have lower levels of ellipsoidal distortion $(\varepsilon=1.28)$ than the linear copolymer micelle $(\varepsilon=1.50)$. In addition, the micelle exhibits a length scale imbalance between the core and corona regions, as observed for the brush tadpole-A copolymer micelle. In contrast to the tadpole-A copolymer micelle, however, the core radius is 1.3 times longer than the thickness of the entire corona region. Furthermore, the large core was found to form with a much higher block chain aggregation number $\left(N_{\mathrm{agg}}=111\right)$ than the core of the tadpole-A copolymer micelle. The contrasting micellization behaviour stems from the linear topology of the hydrophobic block, which is a feature that is also present in the brush linear copolymer analogue. Considering that the brush linear copolymer micelles demonstrated the necessity for a large number of block chains for obtaining a sufficient enthalpy gain to form a stable core, a similar situation is expected in the core formation of the linear hydrophobic block of the tadpole-B copolymer. The comparison of the block chain aggregation numbers of the tadpole-B copolymer micelle and linear copolymer micelle, however, reveals that the necessary number of block chains for a stable core can be greatly reduced when the corona region consists of a cyclic hydrophilic block. The reduced hydrodynamic volume of the cyclic hydrophilic block promotes the formation of a compact and dense corona as opposed to the looser and thicker corona formed by linear hydrophilic block chains. The compact and dense corona is thought to aid in lowering the threshold enthalpy gain necessary for forming a stable core. This particular beneficial element of the cyclic block corona regions is evidenced by the size and interfacial characteristics of the micelle. Despite the relatively larger core radius, the overall micelle size is slightly smaller than that of the tadpole-A copolymer micelle. This feature demonstrates that the cyclic hydrophilic block chain made a greater contribution to reducing the overall micelle size than the cyclic hydrophobic block chain. The core-dense corona interface is sharper than those of the linear and tadpole-A copolymer micelles. The micelle size distribution, however, is broader than that of the tadpole-A analogue. This broader size distribution is mainly attributed to the core consisting of linear hydrophobic block chains. Therefore, the stability of the brush tadpole-B copolymer micelle could be somewhat lower than that of the brush tadpole-A copolymer micelle.

Fourth, the brush linear copolymer micelle exhibits a typical micellar radial density distribution profile, in which the maximum density is located at the centre of the core and the density value gradually decreases through the core region and then drastically reduces through the corona regions (Figure $3 \mathrm{~b}$ ). A similar radial density distribution is observed for the brush tadpole-B copolymer micelle, in which the core consists of linear hydrophobic block chains. These density distribution profiles (namely, aggregation behaviours) could be understood based on the formation characteristics of a prolate ellipsoidal micelle. As a prolate ellipsoid, the micelle is characterized by a polar radius longer than the two equatorial radii. The model analysis has shown that the length of the micelle's polar radius is approximately equal to the length of the linear copolymer chain in a fully extended conformation. It is likely to be that the linear copolymer chains along the polar axis align in stretched conformations. The equatorial radii, in contrast, are shorter than the length of the fully extended linear copolymer chain. This suggests that the linear copolymer chains in the equatorial plane adopt coil-like conformations rather than an extended conformation. Thus, the copolymer chains positioned between the polar axis and the equatorial plane assume intermediate states of stretched and coil-like conformations. Such an unusual phenomenon may originate from the packing of alkyl bristles in the hydrophobic block, which functions as the driving force behind the core formation, and the solvation of hydrophilic block. In the early stages of micelle core formation (that is, the nucleation step of micelle core), for maximal packing of alkyl bristles, lateral alignment of stretched linear hydrophobic block chains occurs along the polar axis of the micelle. As the lateral packing of the hydrophobic chains continues and the initial nucleus of the micelle core grows, the 
Table 3 Structural parameters of micelles of the amphiphilic brush diblock copolymers with various topologies obtained from the quantitative analysis of solution scattering data with a three-phase ellipsoid model

\begin{tabular}{|c|c|c|c|c|}
\hline \multirow{2}{*}{ Structural parameter } & \multicolumn{4}{|c|}{ Amphiphilic brush diblock copolymer } \\
\hline & Linear & Cyclic & Tadpole- $A$ & Tadpole- $B$ \\
\hline$R_{e, \text { micelle }}{ }^{a}(n m)$ & $23.00(1.87)^{\mathrm{b}}$ & $11.55(0.24)$ & $13.60(0.59)$ & $12.70(0.73)$ \\
\hline$r_{\text {core }}{ }^{c}(\mathrm{~nm})$ & $11.60(1.00)$ & $6.10(0.10)$ & $5.20(0.30)$ & $7.20(0.70)$ \\
\hline$t_{f, \text { core }}{ }^{d}(n m)$ & 2.20 & 0.40 & 1.80 & 1.50 \\
\hline$t_{\mathrm{d}, \text { corona }} \mathrm{e}^{\mathrm{e}}(\mathrm{nm})$ & $6.80(1.50)$ & $3.85(0.20)$ & $7.20(0.50)$ & $3.50(0.20)$ \\
\hline$t_{f, \mathrm{~d} . \text { corona }}{ }^{\mathrm{f}}(\mathrm{nm})$ & 2.30 & 0.60 & 1.70 & 1.00 \\
\hline$t_{\text {s.corona }} \mathrm{g}(\mathrm{nm})$ & $4.60(0.50)$ & $1.60(0.10)$ & $1.20(0.10)$ & $2.00(0.10)$ \\
\hline$t_{f, s . c o r o n a}{ }^{h}(n m)$ & 2.0 & 0.20 & 0.30 & 0.40 \\
\hline$\varepsilon^{\mathrm{i}}$ & 1.50 & 0.84 & 0.78 & 1.28 \\
\hline$\xi^{j}(\mathrm{~nm})$ & 1.50 & 1.10 & 0.80 & 0.73 \\
\hline$R_{\mathrm{g}} \mathrm{k}(\mathrm{nm})$ & 21.20 & 8.50 & 9.82 & 10.83 \\
\hline$\rho_{\text {core }}\left(\mathrm{g} \mathrm{cm}^{-3}\right)$ & 1.01 & 0.92 & 0.92 & 1.01 \\
\hline$\rho_{\text {corona }}{ }^{\mathrm{m}}\left(\mathrm{g} \mathrm{cm}^{-3}\right)$ & 0.156 & 0.160 & 0.055 & 0.222 \\
\hline$N_{\text {agg }}{ }^{n}$ & 557 & 41 & 23 & 111 \\
\hline
\end{tabular}

Abbreviation: XR, X-ray reflectivity.

a Radius of micelle in the equatorial direction.

bs.d.

'Radius of core.

dThickness of the fuzzy part (interfaced with the dense corona) of the core.

eThickness of the dense corona.

'Thickness of the fuzzy part (interfaced with the soft corona) of the dense corona.

gThickness of the solvated corona.

Thickness of the fuzzy part (interfaced with solvent) of the solvated corona.

'Ellipsoidicity ratio (polar radius/equatorial radius).

Average correlation length of density fluctuation (that is, blob radius) in the corona part.

kRadius of gyration of micelle.

'Density of core (counting only the nonpolar polymer block chains occupying the core), which is assumed to be the same as that of the corresponding nonpolar homopolymer in films measured by XR analysis.

mDensity of the corona part (counting only the polar polymer block chains occupying the corona), which was estimated from the aggregation number of copolymer chains $N_{\text {agg, }}$, numberaverage molecular weight of the polar block chain and the total volume of corona part.

列 Aggregation number of copolymer chains in a single micelle, which was estimated from the core volume and the assumption that the density of the core composed of the nonpolar block
chains is the same as that of the corresponding nonpolar homopolymer in films measured by XR analysis.

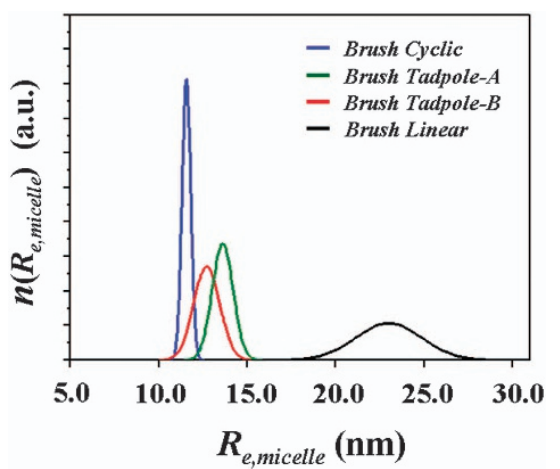

Figure 5 Micelle radius distributions determined from the X-ray scattering data analysis.

hydrophilic block chains' interaction with solvent molecules disturbs the optimal packing behaviour. This is inevitable because upon solvation, the hydrodynamic volume and the dynamic, random chain motion of the hydrophilic block become much greater than those of the hydrophobic block. Hence, to relieve the steric hindrance experienced by the hydrophilic blocks, the hydrophobic blocks begin to adopt a coil-like conformation as the nucleus of the micelle core grows. The adoption of the coil-like conformation induces curvature in the shape of the growing micelle core, and the degree of the coil-like conformation increases until the hydrophobic block chains reach the equatorial plane. This results in the shorter radii of the micelle in the equatorial plane and the overall prolate ellipsoidal shape of the micelle.

Fifth, the brush cyclic copolymer micelle shows a very different radial density distribution from those of the brush linear and tadpoleB copolymer micelles (Figure 3b). The core's centre exhibits a density that is slightly lower $(4.7 \%$ lower) than the maximum value. This density level is retained up to the first half of the core radius from the centre. Beyond that, the density slowly increases through the second half of the core radius, reaching the maximum value at the core-dense corona interface region. Thereafter, the density decreases rapidly through the dense and solvated corona regions. This interesting density profile could be explained by the packing behaviour of alkyl bristles in the absence of the hydrophobic block's chain end. The lack of a chain end forces the hydrophobic block to adopt a folded conformation. Given this topological restriction, the packing of the alkyl bristles is maximized when the hydrophobic block chains take a flat, hairpin-like loop conformation and are stacked on top of each other. In addition, the packing density of the alkyl bristles is low among the bristles positioned along the loop due to the increased spacing and strain stemming from the apex of the loop. As a result, some degree of voids (which are similar to molecular voids) may be introduced in this area. Alkyl bristles positioned along straighter portions of the backbone chain, however, can pack tightly, as they are relieved of the loop-induced strain. The aggregation behaviour therefore can be maximized near the surface of the core, which yields the maximum density at the core-dense corona interface. Such packing behaviour could explain the unusual radial density distribution of the cyclic copolymer micelle and it is a consequence of topological confinement due to the cyclic nature of this copolymer. Another key consequence of the topological confinement effect is the oblate ellipsoidal shape of the micelle. The hairpin-like loop conformation of the hydrophobic blocks promotes a distinct aggregation behaviour driven by vertical stacking that maximizes the lateral packing of the alkyl bristles, especially at the core-dense corona interface. The vertical orientation of the chain stacking establishes the plane of the initial nucleating chains as the equatorial plane of the micelle. As the hydrophobic block chains stack vertically, the large hydrodynamic volume and the dynamic, random chain motion of the hydrophilic block chains disturb the optimal packing behaviour. Hence, the hydrophobic block chains begin to adopt intermediate states between hairpin-like loop and folded coil-like conformations to compensate for the steric destabilization of the hydrophilic blocks as the number of copolymer chains increases. Because of the hydrophobic chains' conformation transition, curvature is induced in the shape of growing micelle core. At the polar axis of the micelle, the chain conformation transition ceases at the folded coil-like conformation. As the folded coil-like conformation is shorter in length than the hairpin-like loop conformation, the polar radius of this micelle becomes the minor axis and the equatorial radii become the major axes. Therefore, the brush cyclic copolymer micelle adopts the shape of an oblate ellipsoid.

Sixth, the brush tadpole-A copolymer micelle apparently exhibits a radial density distribution profile similar to that of the brush cyclic copolymer micelle (Figure 3b). However, the density of the core centre is only $2.5 \%$ lower than the maximum value at the core-dense corona interface. This small density difference within the core is approximately half of that in the cyclic copolymer micelle. Furthermore, the core-dense corona interface in the brush tadpole-A copolymer micelle 
is 4.5 times thicker than that in the cyclic copolymer micelle. The results indicate that regardless of the connection to the linear hydrophilic block, the cyclic hydrophobic block exhibits core formation in the vertical stacking fashion, similar to the cyclic copolymer micelle. Consequently, the core-dense corona interface layer is denser than the core centre, a recurring morphological feature in the cyclic copolymer micelle. The difference in density between the interface and the central region of the core confirms that the extent of topological confinement varies between hydrophobic block chains with fully cyclic and semi-cyclic structures. Overall, these structural characteristics are attributed to the unique topology of the brush tadpole-A copolymer.

Finally, the individual micelle systems also display very unique structural characteristics in the corona regions depending on the molecular topologies. Interestingly, the brush tadpole-B copolymer micelle shows the highest corona density $\rho_{\text {corona }}$, although its $N_{\text {agg }}$ is five times lower than that of the linear copolymer micelle. Overall, the $\rho_{\text {corona }}$ values are in the following decreasing order: tadpole-B copolymer micelle $>$ cyclic copolymer micelle $>$ linear copolymer micelle $\gg$ tadpole-A copolymer micelle. More interestingly, despite having the highest $\rho_{\text {corona }}$ value, the tadpole-B copolymer micelle also exhibits the shortest average correlation length $\xi$ in the corona regions. In contrast, the brush linear copolymer micelle exhibits the largest $\xi$ value, although its $\rho_{\text {corona }}$ value is much higher than that of the brush tadpole-A copolymer micelle. The $\xi$ value is in the following increasing order: tadpole-B copolymer micelle $<$ tadpole-A copolymer micelle $\ll$ cyclic copolymer micelle $<$ linear copolymer micelle. These noteworthy results could be rationalized based on three factors: the topology-induced chain conformation of the hydrophilic block chain, the $N_{\mathrm{agg}}$ value and the overall corona volume. In the brush tadpole-B copolymer micelle, the cyclic hydrophilic block chain demonstrates a smaller hydrodynamic volume in solution than its linear analogue due to the topological confinement effect. Consequently, it has shorter correlation length in the blob contributions. Considering this situation, one may expect a similar topological effect on the corona regions in the cyclic copolymer micelle. However, the cyclic copolymer has a semi-cyclic hydrophilic block chemically linked to the semi-cyclic hydrophobic block and, as previously mentioned, the extent of topological confinement for a block with a semi-cyclic topology is different from that of a block with a fully cyclic topology. The hydrophilic chain segments near the border between the hydrophobic and hydrophilic blocks retain extended conformations, whereas the hydrophilic chain segments positioned further away adopt a coil-like conformation. As a consequence of the dense packing of the hydrophobic blocks at the core-dense corona interface, hydrophilic chain segments experience crowdedness and steric hindrance. To relieve such steric destabilization, the hydrophilic chain segments near the core-dense corona interface retain extended conformations. As the distance from the interface increases, the degree of steric hindrance and crowdedness quickly decreases, and therefore the remaining hydrophilic chain segments take coil-like conformations. Owing to the contribution of the extended hydrophilic chain segments, the semi-cyclic hydrophilic block chain reveals a relatively longer

\section{Nanomicelles in ethanol/water mixture (75/25 in wt\%)}

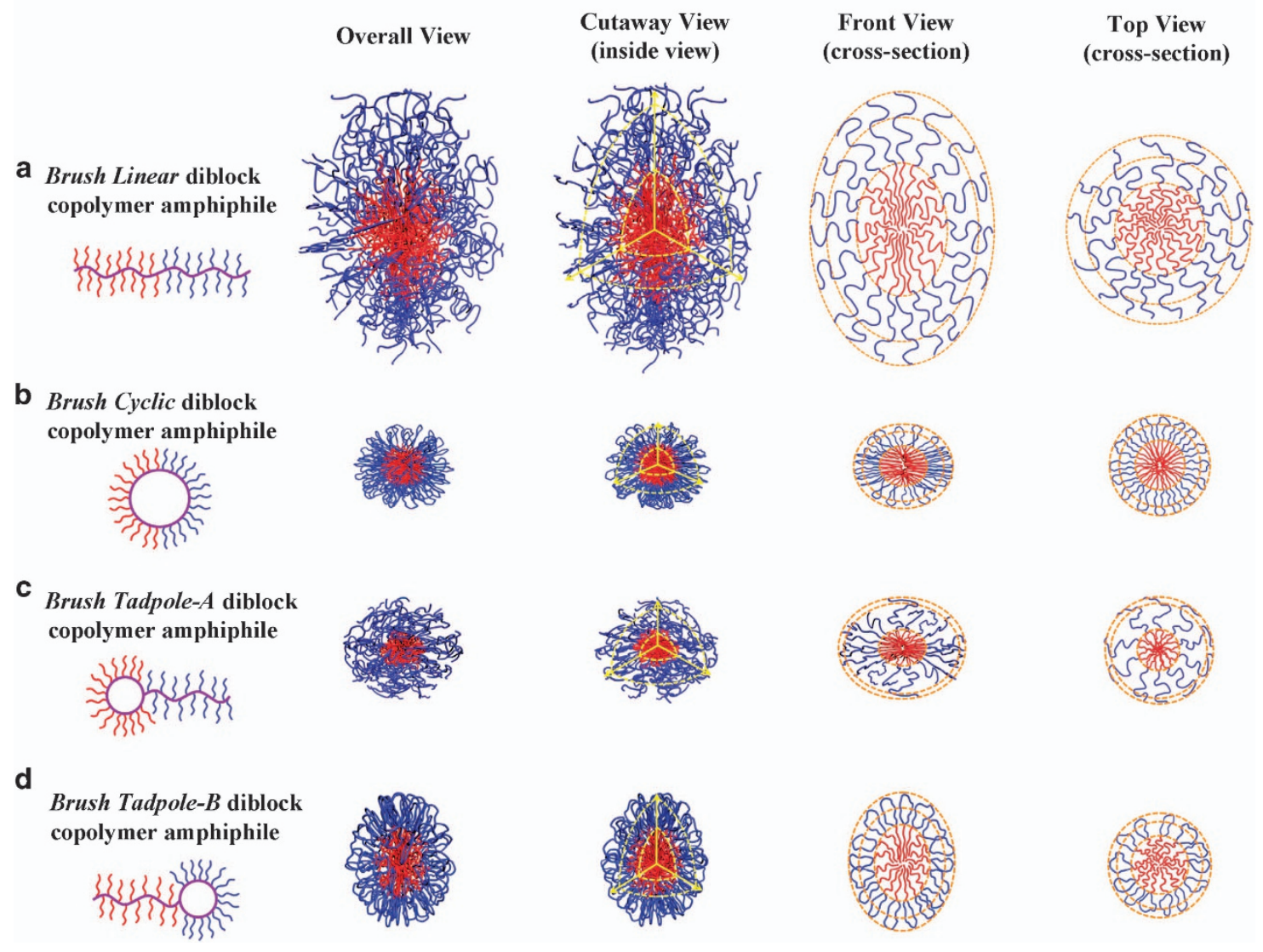

Figure 6 Schematic representations of the micelle structures of amphiphilic brush diblock copolymers with various topologies, which were obtained from the detailed structural parameters determined by the quantitative X-ray scattering data analyses using the three-phase ellipsoid model. 
correlation length in the blob contributions. On the other hand, the linear hydrophilic blocks in the brush tadpole-A copolymer micelle show a relatively short correlation length in the blob contributions. Surprisingly, this correlation length is much shorter than that in the linear copolymer micelle. This difference could be attributed to several factors associated with core formation and molecular topology. For the brush tadpole-A copolymer micelle, the linear block chains are able to favourably interact with solvent molecules in the corona regions simply because of the low number of chains (extremely low $N_{\text {agg }}$ value), and the blob contributions reveal a short correlation length. In contrast, the corona regions in the linear copolymer micelle are crowded with the linear hydrophilic block chains because of the $N_{\text {agg }}$ and $\rho_{\text {corona }}$ values. Under such crowded conditions, the linear block chains can be stabilized by adopting stretched conformations. Chain segments near the core-dense corona interface are forced to be in an extended conformation by the chemical bonds anchoring them to the core interface in a relatively confined space. These two factors cooperatively cause the linear block chains in the corona regions to have a relatively longer correlation length in the blob contributions.

From the aforementioned analysis results and insights, phaseseparated structural models have been schematically constructed for the micelles of amphiphilic brush diblock copolymers with the linear, cyclic, tadpole-A and tadpole-B topologies. The proposed micelle models are shown in Figure 6.

Overall, the quantitative analysis of this study has shown that the micelle formation behaviour of an amphiphilic diblock copolymer is sensitively and precisely controlled by all the aspects of molecular topology. From the results in this study, the absence of backbone chain end groups in an amphiphilic brush diblock copolymer can be rationalized to primarily impact the overall micellization behaviour in both the core and the corona and the structural stability. A hydrophobic brush block with cyclic topology favourably self-assembles, forming most compact and stable core with fewer block chains. The hydrophilic brush block with cyclic topology forms compact corona regions, which helps prevent intermicellar interactions that tend to cause undesired secondary aggregations. Therefore, the stable core aggregation behaviour acts as the primary factor in determining the overall micelle structural stability, whereas the formation of compact corona regions with no intermicellar interactions serves as a secondary factor in controlling the micelle structural stability with a unimodal, narrow size distribution. However, a hydrophobic block with no backbone chain end groups leads to the generation of oblate ellipsoidal micelle because of the semi-cyclic or fully cyclic topology, which is considerably different from the prolate ellipsoidal micellization of the linear analogue possessing a backbone end group and from the spherical micellization that appears very often in the literature.

\section{CONCLUSION}

In this study, the micellization behaviour and structural details of linear, cyclic, tadpole-A and tadpole-B diblock copolymers composed of hydrophobic PDGE and hydrophilic PTEGGE in an equivalent degree of polymerization were investigated by synchrotron X-ray scattering.

All the amphiphilic brush copolymers were found to form micelles in a mixture of ethanol and water $(75 / 25 \mathrm{wt} \%)$. The scattering invariant analysis revealed that the CMC varies from $0.015 \mathrm{wt} \%$ to $0.028 \mathrm{wt} \%$ depending on the molecular topologies. The CMC is in the following increasing order: tadpole-A copolymer $<$ tadpole-B copolymer $\ll$ cyclic copolymer $<$ linear copolymer. The $\mathrm{CMC}$ value could be significantly lowered by the presence of a fully cyclic or semi-cyclic block component.
The structural characteristics of the individual micelle systems were successfully parameterized by the quantitative analysis of X-ray scattering data based on a newly developed three-phase ellipsoidal model method in combination with the IFT analytical scheme. The analysis found that all the copolymers can form micelles with a unimodal size distribution. All the micelles were composed of a core, a dense corona and a solvated corona. The analysis further confirmed that the molecular topology significantly affects micelle structure in all aspects as follows. The brush semi-cyclic and fully cyclic hydrophobic blocks lead to the formation of oblate ellipsoidal micelles with unique density profiles, whereas the brush linear blocks afford prolate ellipsoidal micelles with conventional density profiles. The core size is in the following decreasing order: linear copolymer $\gg$ tadpole- $\mathrm{B}$ copolymer $>$ cyclic copolymer $>$ tadpole-A copolymer. The overall corona thickness is in the following decreasing order: linear copolymer $\gg$ tadpole-A copolymer $>$ tadpole-B copolymer $>$ cyclic copolymer. The aggregation number of copolymer chains is in the following decreasing order: linear copolymer $\gg$ tadpole-B copolymer $\gg$ cyclic copolymer $>$ tadpole-A copolymer. The overall micelle size is in the following decreasing order: linear copolymer $\gg$ tadpole-A copolymer $>$ tadpole-B copolymer $>$ cyclic copolymer. The micelle size distribution is in the following decreasing order: linear copolymer $\gg$ tadpole-B copolymer $>$ tadpole-A copolymer $\gg$ cyclic copolymer. The overall micelle stability is in the following increasing order: linear copolymer $\ll$ tadpole-B copolymer $<$ tadpole-A copolymer $\ll$ cyclic copolymer. From the structural analysis details, it can be concluded that the absence of backbone chain end groups in an amphiphilic brush diblock copolymer is the primary factor affecting the overall micellization behaviour and the structural stability. The brush cyclic hydrophobic block leads to the formation of the most compact and stable micelle core with fewer polymer chains. In addition to the cyclic topology, the presence of hydrophobic bristles reinforces the micelle core stability, as the bristles serve to establish stable packing behaviour driven by the large number of bristles and tight positioning along the polymer. The brush cyclic hydrophilic block drives the formation of compact corona regions, which can prevent intermicellar aggregation. Moreover, the hydrophilic bristles themselves further enhance the compactness of the corona by reducing the crevices between the polymer block chains in the micelle corona via their solvated volumes filling up the gaps. In conjunction with the cyclic topology, hydrophilic bristles function to prevent potential penetration by polymer chains from other micelles and decrease the risk of intermicellar aggregation.

Collectively, all the results of this study provide unique opportunities for designing advanced functional high-performance polymer materials for micelles and realizing their applications in a variety of key scientific and industrial fields (drug delivery, biomedical imaging, foods, cosmetics, smart coatings, photonics, molecular electronics and so on) that would be unattainable by other conventional means.

\section{CONFLICT OF INTEREST}

The authors declare no conflict of interest.

\section{ACKNOWLEDGEMENTS}

This research was supported by the Bio \& Medical Technology Development Program of the National Research Foundation of Korea (NRF) funded by the Ministry of Science and ICT (MSIT) (NRF-2017M3A9F5030930). This work was also supported by the MEXT programme 'Strategic Molecular and Materials Chemistry through Innovative Coupling Reactions' of Hokkaido University, the MEXT Grant-in-Aid for Challenging Exploratory Research (Grant 25620089 and 16K14000), and the MEXT Grant-in-Aid for Scientific 
Research on Innovative Areas 'Advanced Molecular Transformation by Organocatalysts'. BJR received a JSPS Fellowship for Young Scientists from the Japan Society for the Promotion of Science (JSPS). The synchrotron X-ray scattering measurements at the Pohang Accelerator Laboratory were supported by the MSIT, the POSTECH Foundation and POSCO Company.

Author contributions: TS, WJK and MR designed the research and initiated the study. BJR and KSJ prepared the nanomicelles and characterized them in detail. YS, TI and TK synthesized and characterized the materials. BJR, YS, TS, WJK and MR prepared the manuscript. All authors contributed to the discussion. In particular, BJR and YS equally contributed to this work.

\section{PUBLISHER'S NOTE}

Springer Nature remains neutral with regard to jurisdictional claims in published maps and institutional affiliations.

1 Honda, S., Yamamoto, T. \& Tezuka, Y. Topology-directed control on thermal stability: Micelles formed from linear and cyclized amphiphilic block copolymers. J. Am. Chem Soc. 132, 10251-10253 (2010).

2 Laurent, B. A. \& Grayson, S. M. Synthetic approaches for the preparation of cyclic polymers. Chem. Soc. Rev. 38, 2202-2213 (2009).

3 Cooke, J., Viras, K., Yu, G.-E., Sun, T., Yonemitsu, T., Ryan, A. J., Price, C. \& Booth, C. Large cyclic poly(oxyethylene)s: chain folding in the crystalline state studied by Raman spectroscopy, X-ray scattering, and differential scanning calorimetry. Macromolecules 31, 3030-3039 (1998)

4 Cortez, M. A., Godbey, W. T., Fang, Y., Payne, M. E., Cafferty, B. J., Kosakowska, K. A. \& Grayson, S. M. The synthesis of cyclic poly(ethylene imine) and exact linear analogues: an evaluation of gene delivery comparing polymer architectures. J. Am. Chem. Soc. 137, 6541-6549 (2015)

5 Yamamoto, T. \& Tezuka, Y. Cyclic polymers revealing topology effects upon selfassemblies, dynamics and responses. Soft Matter 11, 7458-7468 (2015).

6 Fan, X., Huang, B., Wang, G. \& Huang, J. Synthesis of amphiphilic heteroeight-shaped polymer cyclic-[poly(ethylene oxide)-b-polystyrene]2 via 'click' chemistry. Macromolecules 45, 3779-3786 (2012).

7 Liu, B., Wang, H., Zhang, L., Yang, G., Liu, X. \& Kim, I. A facile approach for the synthesis of cyclic poly(Nisopropylacrylamide) based on an anthracene-thiol click reaction. Polym. Chem. 4, 2428-2431 (2013).

8 Qiu, X.-P., Tanaka, F. \& Winnik, F. M. Temperature-induced phase transition of welldefined cyclic poly(N-isopropylacrylamide)s in aqueous solution. Macromolecules 40, 7069-7071 (2007).

$9 \mathrm{Xu}$, J., Ye, J. \& Liu, S. Synthesis of well-defined cyclic poly(N-isopropylacrylamide) via click chemistry and its unique thermal phase transition behavior. Macromolecules 40 , 9103-9110 (2007)

10 Zhu, X., Zhou, N., Zhang, Z., Sun, B., Yang, Y., Zhu, J. \& Zhu, X. Cyclic polymers with pendent carbazole units: Enhanced fluorescence and redox behavior. Angew. Chem. Int. Ed. 50, 6615-6618 (2011).

11 Di Cola, E., Lefebvre, C., Deffieux, A., Narayanan, T. \& Borsali, R. Micellar transformations of poly(styrene-b-isoprene) block copolymers in selective solvents. Soft Matter 5, 1081-1090 (2009).

12 Giacomelli, F. C., Riegel, I. C., Petzhold, C. L., da Silveira, N. P. \& Štěpánek, P. Internal structural characterization of triblock copolymer micelles with looped corona chains. Langmuir 25, 3487-3493 (2009).

$13 \mathrm{Heo}$, K., Kim, Y. Y., Kitazawa, Y., Kim, M., Jin, K. S., Yamamoto, T. \& Ree, M. Structural characteristics of amphiphilic cyclic and linear block copolymer micelles in aqueous solutions. ACS Macro Lett. 3, 233-239 (2014).

14 Honda, S., Koga, M., Tokita, M., Yamamoto, T. \& Tezuka, Y. Phase separation and selfassembly of cyclic amphiphilic block copolymers with a main-chain liquid crystalline segment. Polym. Chem. 6, 4167-4176 (2015).

15 Lin, Y.-L., Wu, M.-Z., Sheng, Y.-J. \& Tsao, H.-K. Effects of molecular architectures and solvophobic additives on the aggregative properties of polymeric surfactants. J. Chem. Phys. 136, 104905 (2012).

16 Lonsdale, D. E. \& Monteiro, M. J. Synthesis and self-assembly of amphiphilic macrocyclic block copolymer topologies. J. Polym. Sci. A Polym. Chem. 49, 4603-4612 (2011).

17 Magerl, D., Philipp, M., Qiu, X.-P., Winnik, F. M. \& Müller-Buschbaum, P. Swelling and thermoresponsive behavior of linear versus cyclic poly( $\mathrm{N}$-isopropylacrylamide) thin films. Macromolecules 48, 3104-3111 (2015).

18 Minatti, E., Borsali, R., Schappacher, M., Deffieux, A., Soldi, V., Narayanan, T. \& Putaux, J.-L. Effect of cyclization of polystyrene/polyisoprene block copolymers on their micellar morphology. Macromol. Rapid Commun. 23, 978-982 (2002).

19 Ren, J. M., Satoh, K., Goh, T. K., Blencowe, A., Nagai, K., Ishitake, K., Christofferson, A. J., Yiapanis, G., Yarovsky, I., Kamigaito, M. \& Qiao, G. G.
Stereospecific cyclic poly(methyl methacrylate) and its topology-guided hierarchically controlled supramolecular assemblies. Angew. Chem. Int. Ed. 53, 459-464 (2014).

20 Shin, E. J., Jeong, W., Brown, H. A., Koo, B. J., Hedrick, J. L. \& Waymouth, R. M. Crystallization of cyclic polymers: Synthesis and crystallization behavior of high molecular weight cyclic poly(e-caprolactone)s. Macromolecules 44, 2773-2779 (2011).

21 Takeshita, H., Poovarodom, M., Kiya, T., Arai, F., Takenaka, K., Miya, M. \& Shiomi, T. Crystallization behavior and chain folding manner of cyclic, star and linear poly (tetrahydrofuran)s. Polymer 53, 5375-5384 (2012).

22 Williams, R. J., Dove, A. P. \& O'Reilly, R. K. Self-assembly of cyclic polymers. Polym. Chem. 6, 2998-3008 (2015).

23 Zhao, Y., Liu, Y.-T., Lu, Z.-Y. \& Sun, C.-C. Effect of molecular architecture on the morphology diversity of the multicompartment micelles: a dissipative particle dynamics simulation study. Polymer 49, 4899-4909 (2008)

24 Zhu, Y., Gido, S. P., latrou, H., Hadjichristidis, N. \& Mays, J. M. Microphase separation of cyclic block copolymers of styrene and butadiene and of their corresponding linear triblock copolymers. Macromolecules 36, 148-152 (2003).

25 Yue, J., Liu, S., Xie, Z., Xing, Y. \& Jing, X. Size-dependent biodistribution and antitumor efficacy of polymer micelle drug delivery systems. J. Mater. Chem. B 1, 4273-4280 (2013)

26 Scholz, C., lijima, M., Nagasaki, Y. \& Kataoka, K. Polymeric micelles as drug delivery systems: a reactive polymeric micelle carrying aldehyde groups. Polym. Adv. Technol. 9, 768-776 (1998).

27 Danhier, F., Feron, O. \& Préat, V. To exploit the tumor microenvironment: passive and active tumor targeting of nanocarriers for anti-cancer drug delivery. J. Control. Release 148, 135-146 (2010).

28 Matsumura, Y. \& Maeda, H. A new concept for macromolecular therapeutics in cancer chemotherapy: mechanism of tumoritropic accumulation of proteins and the antitumor agent smancs. Cancer Res. 46, 6387-6392 (1986).

29 Isono, T., Satoh, Y., Miyachi, K., Chen, Y., Sato, S., Tajima, K., Satoh, T. \& Kakuchi, T. Synthesis of linear, cyclic, figure-eight-shaped, and tadpole-shaped amphiphilic block copolyethers via t-Bu-P4-catalyzed ring-opening polymerization of hydrophilic and hydrophobic glycidyl ethers. Macromolecules 47, 2853-2863 (2014).

30 Shin, S. R., Jin, K. S., Lee, C. K., Kim, S. I., Spinks, G. M., So, I., Jeon, J.-H., Kang, T. M., Mun, J. Y., Han, S.-S., Ree, M. \& Kim, S. J. Fullerene attachment enhances performance of a DNA nanomachine. Adv. Mater. 21, 1907-1910 (2009).

31 Lee, B., Park, Y.-H., Hwang, Y.-T., Oh, W., Yoon, J. \& Ree, M. Ultralow-k nanoporous organosilicate dielectric films imprinted with dendritic spheres. Nat. Mater. 4, 147-150 (2005).

32 Kim, Y. Y., Ree, B. J., Kido, M., Ko, Y.-G., Ishige, R., Hirai, T., Wi, D., Kim, J., Kim, W. J., Takahara, A. \& Ree, M. High-performance n-type electrical memory and morphology-induced memory-mode tuning of a well-defined brush polymer bearing perylene diimide moieties. Adv. Electronic Mater. 1, 1500197 (2015).

33 Bolze, J., Ree, M., Youn, H. W., Chu, S.-H. \& Char, K. Synchrotron X-ray reflectivity study on the structure of templated polyorganosilicate thin films and their derived nanoporous analogues. Langmuir 17, 6683-6691 (2001).

34 Parratt, L. G. Surface studies of solids by total reflection of X-rays. Phys. Rev. 95, 359-369 (1954).

35 Guinier, A. \& Fournet, G. Small Angle Scattering X-Ray, Wiley: New York, (1955).

36 Glatter, O. J. A new method for the evaluation of small-angle scattering data. Appl. Crystallogr. 10, 415-421 (1977).

37 Pedersen, J. S. \& Gerstenberg, M. C. Scattering form factor of block copolymer micelles. Macromolecules 29, 1363-1365 (1996).

38 Pedersen, J. S. Analysis of small-angle scattering data from colloids and polymer solutions: modeling and least-squares fitting. Adv. Colloid Interface Sci. 70, 171-210 (1997)

39 Rathgeber, S., Monkenbusch, M., Kreitschmann, M., Urban, V. \& Brulet, A. Dynamics of star-burst dendrimers in solution in relation to their structural properties. J. Chem. Phys. 117, 4047-4062 (2002).

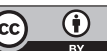

This work is licensed under a Creative Commons Attribution 4.0 International License. The images or other third party material in this article are included in the article's Creative Commons license, unless indicated otherwise in the credit line; if the material is not included under the Creative Commons license, users will need to obtain permission from the license holder to reproduce the material. To view a copy of this license, visit http:// creativecommons.org/licenses/by/4.0/

(C) The Author(s) 2017

Supplementary Information accompanies the paper on the NPG Asia Materials website (http://www.nature.com/am) 